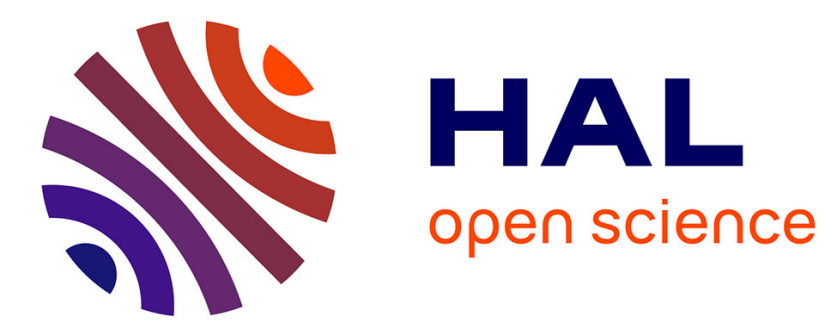

\title{
Wiener-Askey and Wiener-Haar expansions for the analysis and prediction of limit cycle oscillations in uncertain nonlinear dynamic friction systems
}

Lyes Nechak, Sébastien Berger, Evelyne Aubry

\section{- To cite this version:}

Lyes Nechak, Sébastien Berger, Evelyne Aubry. Wiener-Askey and Wiener-Haar expansions for the analysis and prediction of limit cycle oscillations in uncertain nonlinear dynamic friction systems. Journal of Computational and Nonlinear Dynamics, 2013, 9 (2), pp.1-12. 10.1115/1.4024851 . hal01027766

\section{HAL Id: hal-01027766 \\ https://hal.science/hal-01027766}

Submitted on 27 May 2020

HAL is a multi-disciplinary open access archive for the deposit and dissemination of scientific research documents, whether they are published or not. The documents may come from teaching and research institutions in France or abroad, or from public or private research centers.
L'archive ouverte pluridisciplinaire HAL, est destinée au dépôt et à la diffusion de documents scientifiques de niveau recherche, publiés ou non, émanant des établissements d'enseignement et de recherche français ou étrangers, des laboratoires publics ou privés.

\section{(c)(1)}

Distributed under a Creative Commons Attribution| 4.0 International License 


\section{Wiener-Askey and Wiener-Haar Expansions for the Analysis and Prediction of Limit Cycle Oscillations in Uncertain Nonlinear Dynamic Friction Systems}

\begin{abstract}
This paper is devoted to the robust modeling and prediction of limit cycle oscillations in nonlinear dynamic friction systems with a random friction coefficient. In recent studies, the Wiener-Askey and Wiener-Haar expansions have been proposed to deal with these problems with great efficiency. In these studies, the random dispersion of the friction coefficient is always considered within intervals near the Hopf bifurcation point. However, it is well known that friction induced vibrations - with respect to the distance of the friction dispersion interval to the Hopf bifurcation point-have different properties in terms of tansient, frequency and amplitudes. So, the main objective of this study is to analyze the capabilities of the Wiener-Askey (general polynomial chaos, multielement generalized polynomial chaos) and Wiener-Haar expansions to be efficient in the modeling and prediction of limit cycle oscillations independently of the location of the instability zone with respect to the Hopf bifurcation point.
\end{abstract}

Keywords: uncertain nonlinear systems, nonlinear dynamic systems, mode coupling instability, random uncertainty, limit cycle oscillation, friction-induced vibration, generalized polynomial chaos-multi-element generalized polynomial chaos, wiener-haar

\author{
Lyes Nechak ${ }^{1}$ \\ MIPS Laboratory, \\ 12, Rue des Frères Lumière, \\ Mulhouse 68093, France \\ e-mail: lyes.nechak@uha.fr
}

\author{
Sébastien Berger \\ MIPS Laboratory \\ 12, Rue des Frères Lumière, \\ Mulhouse 68093, France \\ e-mail: sebastien.berger@uha.fr \\ Evelyne Aubry \\ MIPS Laboratory, \\ 12, Rue des Frères Lumière, \\ Mulhouse 68093, France \\ e-mail: evelyne.aubry@uha.fr
}

\section{Introduction}

Great interest has been attached to self-excited friction induced vibrations due to their high importance in broad varieties of engineering applications, such as the aeronautic, railway and automotive fields. Braking, clutch, and wiping systems are well known examples of self-excited friction systems where different unforced vibrations may be observed [1-7]. For example, in the automotive field, self-excited friction induced vibrations occur during a braking event and are identified by means of quasimonochromatic sounds emitted at high frequencies $(>10 \mathrm{Khz})$ named braking squeal. Numerous recent studies continue to be devoted to the development of approaches and tools for the prediction of brake squeal noise; see Ref. [8] where nonlinear statistical analysis is used or in Ref. [9] where brake squeal is treated as a chaotic phenomenon.

Another example of friction induced vibrations concerns the sliding phase of clutch engagement [2,3,7]. They lead to audible disturbance defined as clutch squeal noise [5].

Several studies have focused on the mechanisms which are responsible for self-excited friction induced vibrations. So, various mechanisms have been defined to explain the self-friction-induced vibration phenomenon. They are classified into two main families. The first one is related to the tribological aspects of friction systems and includes the stick-slip and speed dependent friction force mechanisms, while the second family is related to geometrical and structural properties and includes the so-called sprag-slip and mode coupling mechanisms [7,10-15]. The stick-slip and speed dependent friction force rely on changes in the friction coefficient according to the relative sliding speed between two bodies in contact. The stick-slip received greater interest. It characterizes instabilities as a consequence of a low sliding speed phenomenon caused when the static friction coefficient is higher than the dynamic friction coefficient. The stick-slip was shown to be relevant in modeling friction induced instabilities such as low frequency brake vibrations [1,16-21]. Nevertheless, numerous studies have pointed out that a decrease in the friction coefficient is not sufficient to explain other types of friction-induced vibrations [12,22-24]. For example, squeal noise, known to be a relatively high frequency phenomenon (up to several Khz), may occur even when the friction coefficient is almost constant versus sliding speed. The tribological properties are not the only cause of instabilities. In this context, the structural and geometrical based mechanisms were proposed, namely the sprag-slip and mode coupling, by which friction-induced oscillations are generated with a constant friction coefficient. The sprag slip was presented in 1961 by Spurr who gave a purely structural interpretation of the selfexcited friction induced vibrations [12]. A more developed theory describes instabilities as geometrically induced or kinematic constraint instability. D'Souza and Dweib have shown analytically that friction is responsible for the presence of a coupling term between modes, which induces the destabilization of a stationary state [23]. The same conclusion appears in numerous other studies. Hoffmann proposes a two degree of freedom system in which the friction-induced vibrations are explained by a mode coupling phenomenon defined by a coalescence of the system's modes which occurs with a constant friction coefficient [25]. In Meziane and Baillet's study, authors have studied vibrations generated at contact with friction for two different applications: one of which is to analyze friction-induced vibrations of a beam-on-beam system in contact with friction. The authors have shown the 
requirement to have the complementarity between a linear analysis of the stability and a nonlinear temporal analysis to well characterize friction induced vibrations [26].

In this paper, emphasis is placed on friction-induced vibrations issued from a mode coupling phenomenon; thus, a constant friction coefficient is considered. However, numerous recent studies focus on the mode coupling instability with an uncertain friction coefficient for the robust analysis of dynamic behaviors of dry friction systems. In fact, dynamic behaviors of dry friction systems have been demonstrated to be very sensitive to design parameters in particular to the friction coefficient. This sensitivity appears in changes of stability properties and thus in the amplitude of the friction induced vibrations. Otherwise, friction has been shown to admit dispersions which may be due to the manufacturing process. So, it is necessary to take this uncertainty into account to ensure robustness of the analysis of friction systems and thus robustness of design of this class of systems. Ragot et al. propose the interval theory to surround the dispersion of friction induced vibrations (limit cycle oscillations (LCOs)) due to the uncertainty of the friction coefficient. The method used has been shown to be inefficient due to the pessimism phenomenon which induces the divergence of the envelopes of limit cycle amplitudes [27]. The polynomial chaos formalism has been proposed as a more efficient alternative to take account of the random uncertainty of the friction coefficient in the study of the dynamic behavior of friction systems, [28,29]. In the Lee et al. study [28], the polynomial chaos formalism is used to analyze a three dimensional nonlinear dry friction model at the wheel-rail contact. The authors show that the probability density function (PDF) of the friction coefficient is affected by the stochastic variation of dynamic parameters as the lateral displacement of the wheel and the rail roughness. The probabilistic dispersion of the friction coefficient was taken into account in the robust analysis of the dynamic behavior of a drum brake developed in the Nechak et al. study [29]. In a first step, the intrusive scheme of the polynomial chaos was combined with the direct Lyapunov approach to analyze the stability. In a second step, the intrusive and nonintrusive schemes were exploited to estimate the maximum amplitudes for the limit cycle oscillations when the system was submitted to flutter instabilities. The nonintrusive schemes proved more efficient than the intrusive one. The robust stability of the same system has been analyzed using a nonintrusive scheme of the generalized polynomial chaos in a new study of Nechak et al. [30]. The main idea is to express system's eigenvalues using GPC expansions. The indirect Lyapunov approach is then applied to analyze the stability through the Monte Carlo procedure or solutions of nonlinear optimization problems. In other studies [31,32], Nechak et al. have shown the deficiency of the generalized polynomial chaos (GPC) formalism in the modeling and prediction of long time self-excited friction induced vibrations. This conclusion can be found in other studies related to flutter in aerodynamic systems [33]. The multielement GPC method and the Wiener-Haar expansion have been shown to be more efficient tools to well predict long time self-excited friction induced vibrations [31,32]. In these studies, the random dispersion of the friction coefficient has always been considered within intervals near the Hopf bifurcation. However, it is well known that friction induced vibrations-with respect to the distance of the friction dispersion interval to the Hopf bifurcation point-have different properties in terms of transient, frequency and amplitudes. The main objective of this present study is to analyze the abilities of the GPC, multielement generalized polynomial chaos (ME-GPC), and the Wiener-Haar expansions to well model and predict limit cycle oscillations. In other words, this paper deals with robustness of Wiener-Askey and Wiener-Haar expansions with respect to the location of the friction uncertainty in the flutter instability. It gives an evaluation of how efficiency and accuracy of Wiener-Askey and WienerHaar in the modeling and prediction of LCOs are dependent on the distance of the flutter zones from the Hopf bifurcation point. This evaluation helps to perform a comparison between both
Wiener-Askey and Wiener-Haar expansions which; consequently, help to select the well suitable expansion for the modeling and prediction of friction-induced vibrations. The final goal is to well estimate the amplitude of limit cycle oscillations and its dispersion corresponding to the random dispersion of the friction coefficient. Otherwise, this study completes results of previous studies [31,32] devoted to the modeling and prediction of long time friction-induced vibrations. This paper is organized as follows. The generalized polynomial chaos and the ME-GPC method formalisms are presented in Secs. 2 and 3 followed by a description of the Wiener-Haar expansion in Sec. 4. Followed by the results of the analysis presented in Sec. 5. The friction based mechanism used is described, its stability is investigated, and its dynamic behavior analyzed. All the results are compared to the referential Monte Carlo (MC) method. Finally, the conclusion is given in Sec. 6.

\section{Generalized Polynomial Chaos}

Let $(\Omega, \beta, \operatorname{Pr})$ be a probability space where $\Omega$ is the sample space of the random events $\omega, \beta$ is the $\sigma$-algebra of the subsets of $\Omega$ and $\operatorname{Pr}: \beta \rightarrow[0,1]$ is the probability measure. Let $\xi(\omega):(\Omega, \beta) \rightarrow(\mathbb{R}, \Im)$ be an $\mathbb{R}$-valued continuous random variable, where $\Im$ is the $\sigma$-algebra of Borel subsets of $\mathbb{R}$. For convenience reasons, $\xi$ is used to represent $\xi(\omega)$.

Let

$$
\begin{array}{r}
f: \mathbb{R}^{n} \times \mathbb{R} \rightarrow \mathbb{R}^{n} \\
(x, \mu) \rightarrow f(x, \mu)
\end{array}
$$

such that

$$
\dot{x}(t)=f(x(t), \mu)
$$

be a nonlinear dynamic system where $f$ is a smooth nonlinear vector field, $x(t) \in \mathbb{R}^{n}$ is the state vector and $\mu$ is a real parameter supposed to be uniformly distributed within a given interval $[a$, $b$ ]. $\mu$ is then as random function in the standard variable $\xi$ uniformly distributed within $[-1,1]$,

$$
\mu(\xi)=((a+b) / 2)+((b-a) / 2) \xi
$$

As a consequence of the random character of parameter $\mu$, state variables $x_{i}(t)$ become stochastic processes $x_{i}(t, \xi)$ depending on the time $t$ and the uncertainty modeled by $\xi$.

From the GPC theory [34-38], it is possible to express a second order (with finite variance) process $x_{i}(t, \xi)$ in a truncated orthogonal Legendre polynomial function series such as

$$
x_{i}(t, \xi) \approx \sum_{j=0}^{P} \bar{x}_{i, j} L_{j}(\xi)
$$

$\bar{x}_{i, j}(t)$ are the stochastic modes of the stochastic process $x_{i}(t, \xi)$ with $(i=1, \ldots, n)$ and $L_{j}$ are the Legendre polynomials which are known to be orthogonal within the interval $[-1,1]$ with respect to the constant density function $W(\xi)=1 / 2$ of the uniform variable $\xi[37,38]$.

Using the truncated Legendre polynomial expansion requires the computing of the stochastic modes $\bar{x}_{i, j}(t)$. Intrusive and nonintrusive schemes are defined in this perspective [39-41]. For nonlinear systems with hard nonlinearities and a high number of degrees of freedom, the nonintrusive schemes (such as the regression and the nonintrusive spectral projection methods) are shown to be more efficient than the intrusive scheme in the prediction of short time friction induced vibrations [29]. Indeed, with the nonintrusive scheme, computing the stochastic modes only needs some calculations of the system responses for a set of particular samples of the random variable which models the uncertain parameter. 
This is not the case for the intrusive scheme in which the stochastic modes are calculated from the solution of deterministic differential equations obtained from the Galerkin projection of the uncertain system onto the generalized polynomial chaos basis [29]. So, only nonintrusive methods, in particular the regression technique, are considered in this study. Details on the regression method can be found in several studies as $[29,41]$.

\section{Multi-Element Generalized Polynomial Chaos Approach}

Let $(\Omega, \beta, \operatorname{Pr})$ be a probability space where the main step in the ME-GPC based method is to divide the random space into $m$ nonintersecting elements [38]. Here, the random space is defined by the interval $B=[-1,1]$ in which the standard uniform variable $\xi$ is defined. So, the decomposition $D$ of $B$ is considered, as follows:

$$
D=\left\{\begin{array}{l}
B_{k}=\left[a_{k} b_{k}\right), k=1, \ldots, m \\
B=\bigcup_{k=1}^{m} B_{k} \\
B_{k} \cap B_{j}=\emptyset j \neq k
\end{array}\right.
$$

The indicator random variables $Z_{k}$ is defined with respect to the previous decomposition of $B$,

$$
Z_{k}= \begin{cases}1 & \text { if } \xi \in B_{k} \\ 0 & \text { otherwise }\end{cases}
$$

Consequently, it is shown in Ref. [42], that $\bigcup_{k=1}^{m} Z_{k}^{-1}(1)$ is a decomposition of the $\Omega$ space where

$$
\left\{\begin{array}{l}
\Omega=\bigcup_{k=1}^{m} Z_{k}^{-1}(1) \\
Z_{k}^{-1}(1) \cap Z_{j}^{-1}(1)=\emptyset, \quad j \neq k
\end{array}\right.
$$

In each element $B_{k}$, a local random uniform variable $\zeta_{k}: Z_{k}^{-1}(1) \mapsto B_{k}$, is defined with the conditional probability func$\operatorname{tion} f_{\zeta_{k}}$

$$
f_{\zeta_{k}}=\frac{1}{2^{d} \operatorname{Pr}\left(Z_{k}=1\right)}, \quad k=1, \ldots, m
$$

and

$$
\operatorname{Pr}\left(Z_{k}=1\right)=\frac{b_{k}-a_{k}}{2}
$$

In practice, the local variables $\zeta_{k}$ are expressed in terms of independent random uniform variables $\bar{\xi}_{k(k=1, \ldots m)}$ in $[-11]$ so the Legendre polynomials can be exploited.

$$
\zeta_{k}=\frac{b_{k}-a_{k}}{2} \bar{\xi}_{k}+\frac{b_{k}+a_{k}}{2}
$$

Let $x_{i, k}\left(t, \bar{\xi}_{k}\right)$ be the random process corresponding to the $k$ th element and to the $i$ th state variable of the system, Eq. (1). Its expansion in the Legendre polynomial is given by the following expression:

$$
x_{i, k}\left(t, \bar{\xi}_{k}\right) \approx \sum_{j=0}^{P} \bar{x}_{i, k, j}(t) L_{j}\left(\bar{\xi}_{k}\right), \quad i=1, \ldots, n
$$

The global approximation of the stochastic process $x_{i}(t, \xi)$ is given by

$$
x_{i}(t, \xi) \approx \sum_{k=1}^{m} \sum_{j=0}^{P} \bar{x}_{i, k, j}(t) L_{k, j}\left(\bar{\xi}_{k}\right) Z_{k}
$$

\section{Wiener-Harr Chaos Expansion}

The Wiener-Haar chaos formalism is a generalization of the well-known Haar wavelets expansion to represent random processes. It states the possibility to write a square integrable random function using Haar wavelet series weighted by the generalized Fourier coefficients, also known as detail coefficients. The Wiener-Haar chaos expansion helps to approximate the localized behavior of stochastic processes in the random dimension. This is performed with the decomposition of the random dimension by the Haar wavelets. The stochastic character of the decomposed stochastic process is concentrated in the orthonormal basis generated by the Haar wavelets while the deterministic character is modeled by the detail functions which, in this case, are functions in time named the generalized Fourier coefficients. In the sequel, a brief presentation of the Wiener-Haar chaos expansion is given. A more detailed description of this tool can be found in Refs. [32,33].

The Scaling function $\phi(z)$ and Haar wavelets $\psi(z)$ are piecewise continued functions defined, respectively, by

$$
\begin{gathered}
\phi(z)= \begin{cases}1 & 0<z<1 \\
0 & \text { otherwise }\end{cases} \\
\psi(z)= \begin{cases}1, & 0 \leq z<0.5 \\
-1 & 0.5 \leq z<1 \\
0 & \text { otherwise }\end{cases}
\end{gathered}
$$

Translated and dilated versions of the scaling Haar function and the Haar wavelet function are given as

$$
\begin{aligned}
\phi_{k}^{j}(z) & =2^{j / 2} \phi\left(2^{j} z-k\right) \\
\psi_{k}^{j}(t) & =2^{j / 2} \psi\left(2^{j} t-k\right)
\end{aligned}
$$

Consider the nonlinear differential equation given by the system, Eq. (1), in which the uncertain parameter $\mu$ is modeled by the uniform variable $\xi$ within the interval $[-1,1]$. To extend the use of Haar wavelets from the unit interval $[0,1]$ to non unit interval $[a, b] \subset \mathbb{R}$ (here, $[a b]$ is the Legendre interval $[-1,1]$ ), it is necessary to set

$$
\bar{\psi}_{k}^{j}(\xi)=\psi_{k}^{j}(\operatorname{Pr}(\xi))
$$

such as

$$
\bar{\psi}_{k}^{j}(y)=\psi_{k}^{j}(z)
$$

with $z=T(y)$ where $y$ is a realization of the uniform variable $\xi, z$ is a realization of a uniform variable in the unit interval $[0,1]$ and $T$ is strictly increasing on $[a b]$ so that $z=T(y)$ is one-to-one and invertible. With the orthonormality condition it can be written that

$$
\int_{0}^{1} \psi_{k}^{j}(z) \psi_{m}^{l}(z) d z=\int_{a}^{b} \bar{\psi}_{m}^{l}(y) \bar{\psi}_{m}^{l}(y) d T(y)=\delta_{j l} \delta_{k m}
$$

The latter expression means that the wavelets $\left\{\psi_{k}^{j}(\xi)\right\}$ are orthonormal with respect to the distribution of $\xi$ and form a multi resolution of the space of second order random processes on $[a, b]$ with continuous distributions.

The Wiener-Haar chaos expansion states the possibility to approximate a second order stochastic process $x_{i}(t, \xi), i=1, \ldots, n$, with the Haar wavelet series expansion.

$$
x_{i}(t, \xi) \approx \tilde{x}_{i, 0}^{0}(t)+\sum_{j=0}^{J} \sum_{k=0}^{2^{j}-1} \hat{x}_{i, k}^{j}(t) \psi_{k}^{j}(\operatorname{Pr}(\xi))
$$




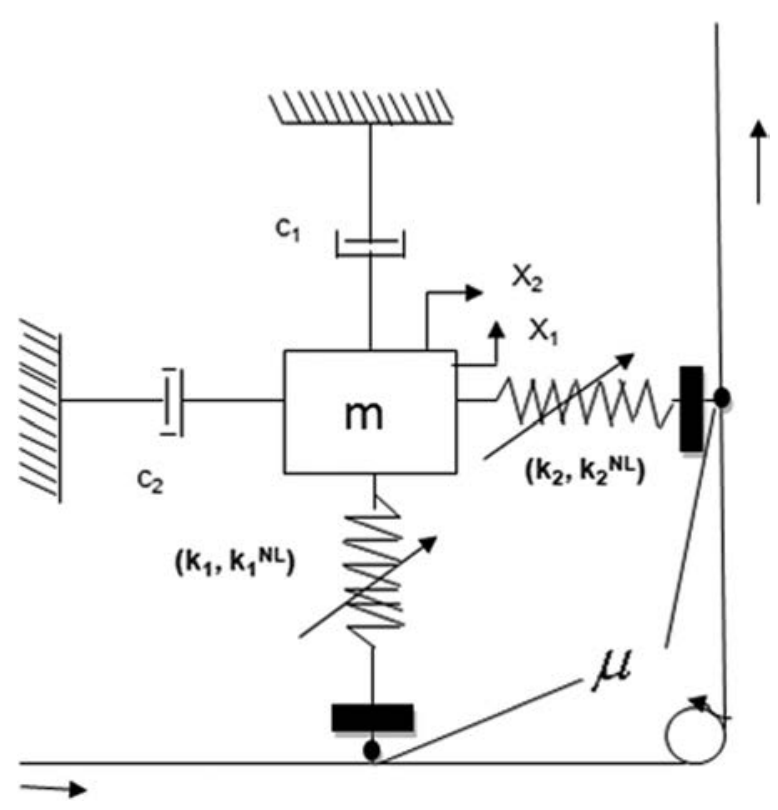

Fig. 1 Hultèn system

where $\tilde{x}_{i}^{0}(t)$ is the instantaneous mean value of the stochastic process $x_{i}(t, \xi)$ such that

$$
\tilde{x}_{i, 0}^{0}(t)=\left\langle x_{i}(t, \xi)\right\rangle=\int_{0}^{1} x_{i}\left(t, T^{-1}(z)\right) \phi_{0}^{0}(z) d z
$$

and the generalized Fourier coefficients are defined by the following integrals

$$
\hat{x}_{i, j}^{k}(t)=\int_{0}^{1} x_{i}\left(t, T^{-1}(z)\right) \psi_{j}^{k}(z) d z
$$

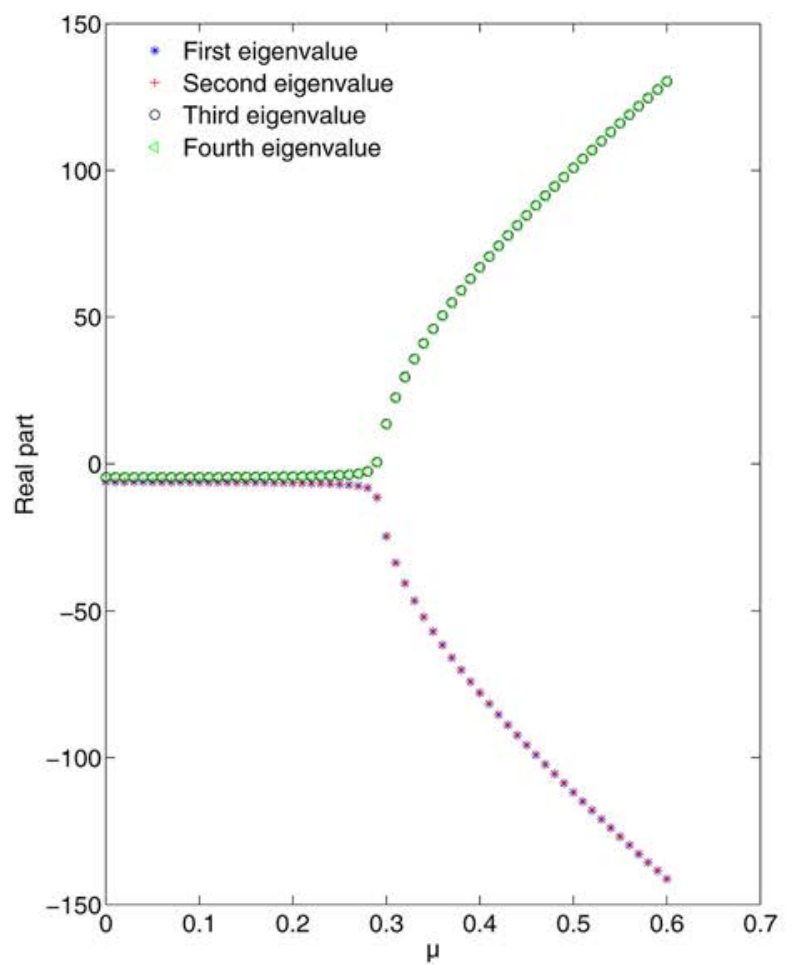

The truncation order of the expansion $J$ defines the resolution level needed to keep the scales which contain significant energy of the approximated process. Like the GPC expansion, the problem is related to the computation of the generalized Fourier coefficients, Eq. (21), for $j=0, \ldots, J$. Numerical integration methods (such as the Monte Carlo, Simpson, and trapezoidal methods) can be used for this aim. However, they are too costly since they need a high number of realizations of the stochastic process $x_{i}\left(t, T^{-1}(z)\right)$ to ensure reasonable accuracy. The Mallat algorithm is a more efficient tool for this computation. Only this algorithm is used in this paper to compute the generalized Fourier coefficients. More details about this result can be founded in the recent work [43].

\section{Application}

As mentioned in Sec. 1, we focus on self-excited friction induced vibrations due to the mode coupling phenomenon. From the latter, self-friction induced oscillations may occur even with a constant friction coefficient $[2,3,12,23,44]$. In these same studies, it has been shown that a two degree-of-freedom model may be sufficient to investigate the mode coupling instability in mechanical friction systems. For instance, Hultèn [45] has defined a two degree-of-freedom model to analyze squeal vibrations. He has demonstrated that the model used is representative of the dynamic behavior of braking systems. Consequently, the same model has been considered in numerous other studies such as the robust damping factor in self-exciting friction mechanism [46] and to analyze the mode coupling instability and its dependency on system parameters including damping [44]. Hultèn has also been considered in Nechak et al. studies [29-32] in the context of robust stability analysis and the prediction of short and long time friction induced vibrations. For all these considerations, the Hultèn model with the same variables and parameters values used in previous studies [29-32] is considered, but in the particular framework which consists of the analysis of efficiency and accuracy of Wiener-Askey and Wiener-Haar expansions in the modeling and prediction of self-excited friction induced vibrations in different flutter instability zones. A description of the Hultèn system is summed up below.

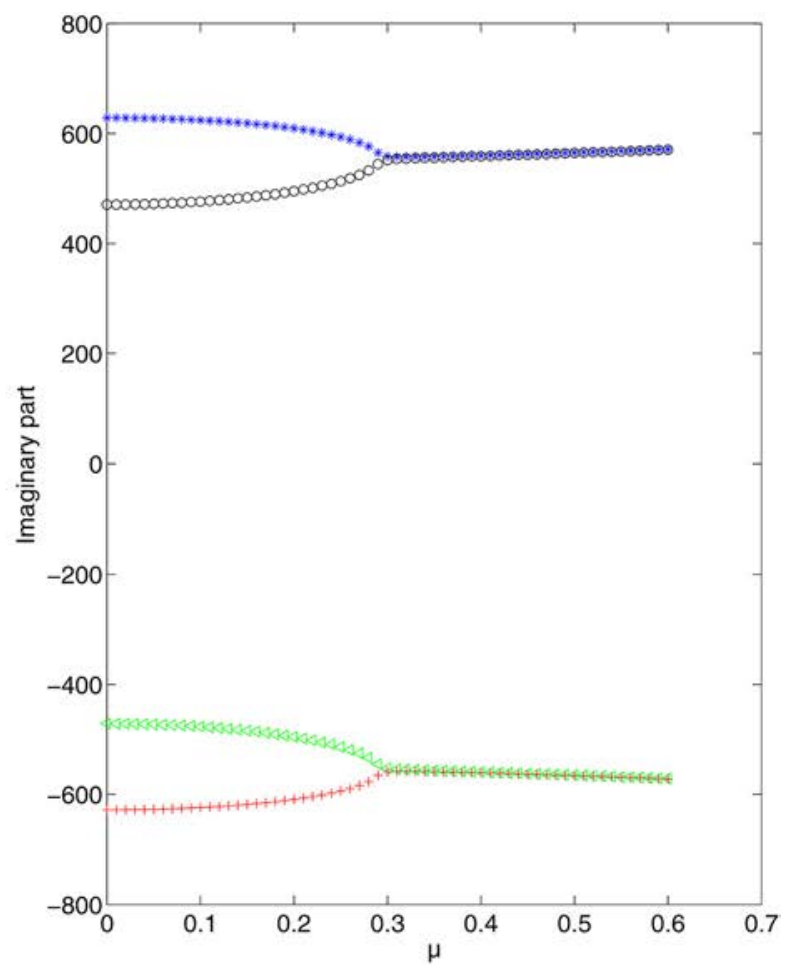

Fig. 2 Evolution of real and imaginary parts of system eigenvalues 
(a) $X$, for $\mu=0.3$
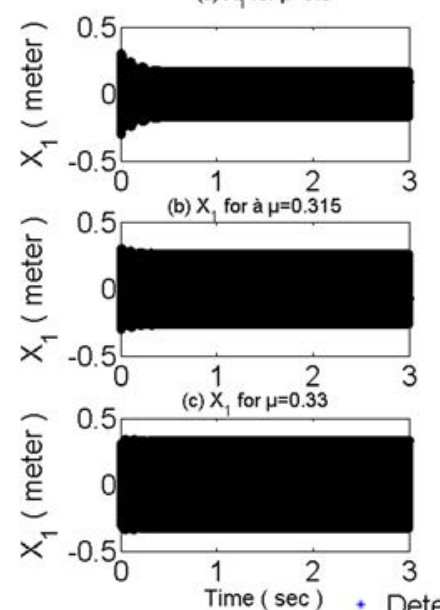

Zoom on $X_{1}$ at $t=0.715 \mathrm{sec}$
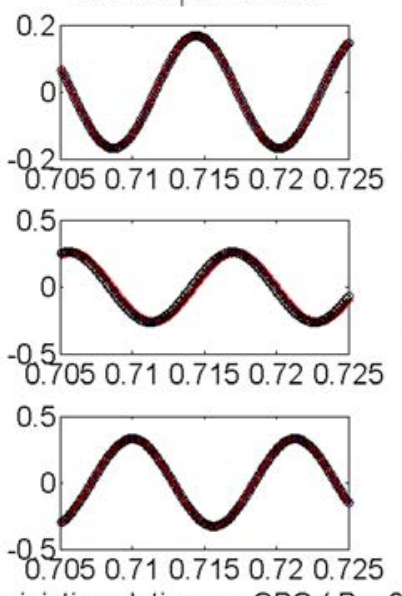

Zoom on $X_{1}$ at $t=2.92 \mathrm{sec}$
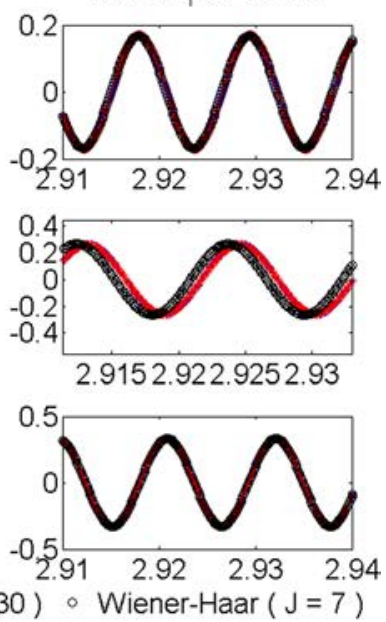

Fig. 3 Realization of the displacement $x_{1}(t, \xi)$ for particular samples of the friction coefficient $\mu$
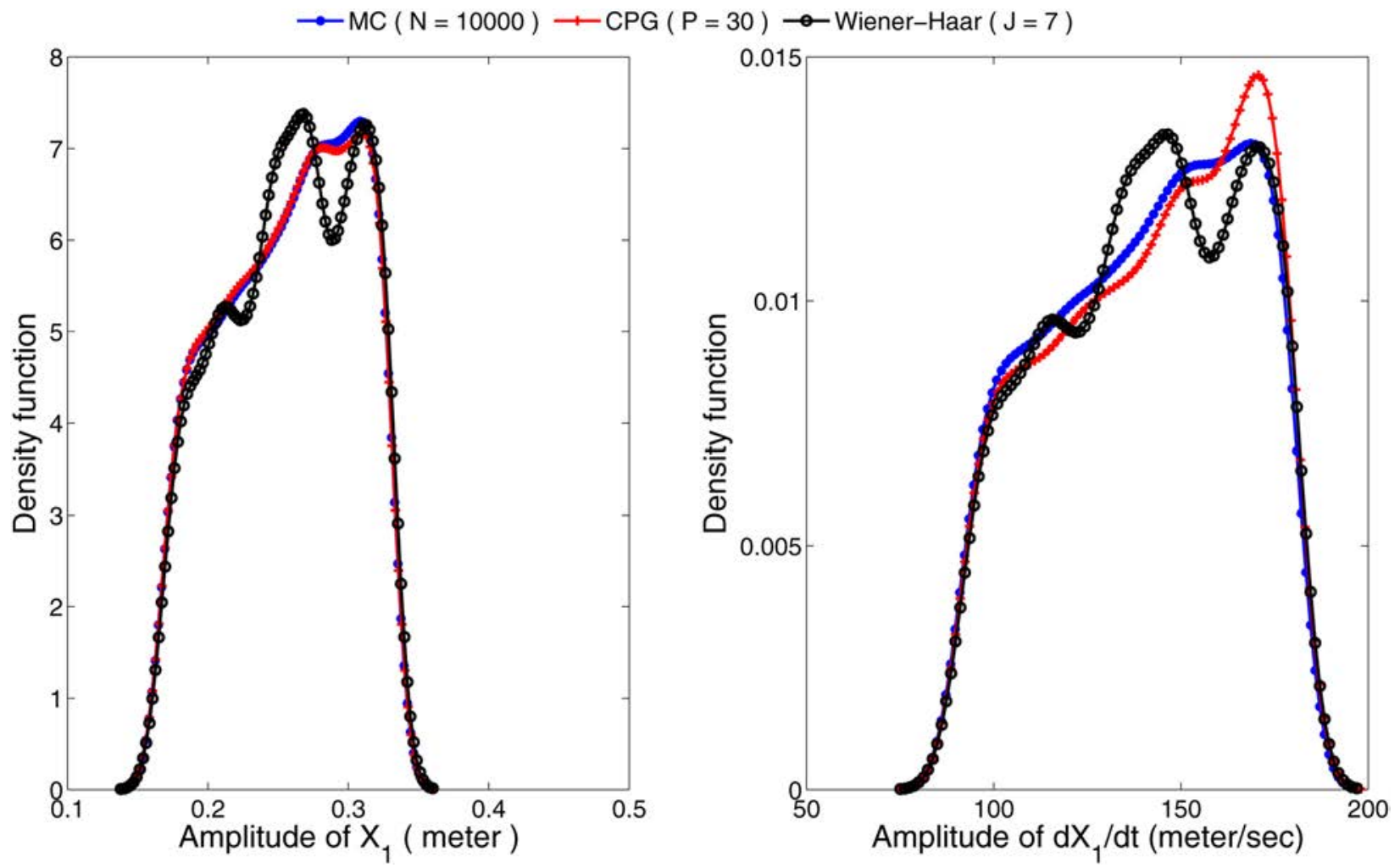

Fig. 4 Density functions of amplitudes corresponding to the friction dispersion near the Hopf bifurcation point

5.1 System Description. The system consists in a mass $M$ held against a moving band; the contact between the mass and the band is modeled by two plates supported by two different springs. For simplicity's sake, it is assumed that the mass and

Table 1 Statistics of the amplitude of $X_{1}$ estimated with the GPC and the Wiener-Haar expansion near the Hopf bifurcation

\begin{tabular}{lcccc}
\hline \hline & $\begin{array}{c}\text { Mean } \\
\text { value }\end{array}$ & $\begin{array}{c}\text { Standard } \\
\text { deviation }(\text { Std) }\end{array}$ & Min & Max \\
\hline MC & 0.2571 & 0.0465 & -0.3309 & 0.3309 \\
GPC $(P=30)$ & 0.2570 & 0.0465 & -0.3309 & 0.3309 \\
Wiener-Haar $(J=7)$ & 0.2582 & 0.0462 & -0.3309 & 0.3309 \\
\hline \hline
\end{tabular}

band surfaces always keep in contact. This assumption is justified by a preload applied to the system. The contact can be expressed by two cubic stiffnesses. Damping is integrated as shown in Fig. 1.

Table 2 Statistics of the amplitude of $d X_{1} / \mathrm{dt}$ estimated with the GPC and the Wiener-Haar expansion near the Hopf bifurcation

\begin{tabular}{lcccc}
\hline \hline & Mean & Std & Min & Max \\
\hline MC & 138.6277 & 28.9007 & -180.9520 & 180.9983 \\
GPC $(P=30)$ & 141.6692 & 25.8141 & -181.2867 & 181.3285 \\
Wiener-Haar $(J=7)$ & 140.9610 & 25.3625 & -180.9766 & 180.9760 \\
\hline \hline
\end{tabular}




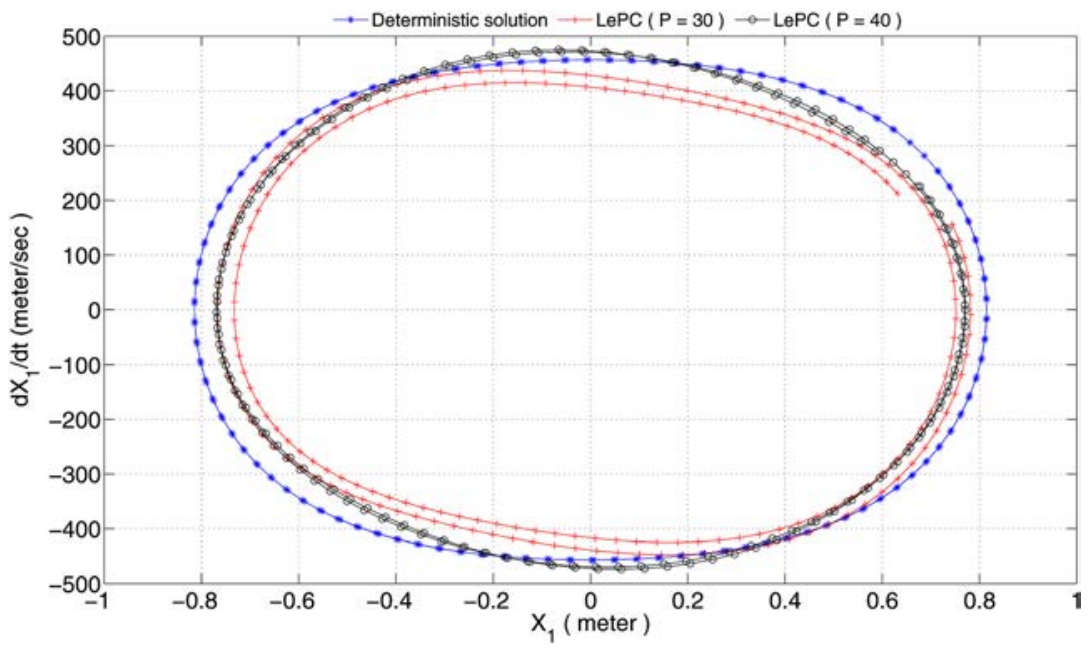

Fig. 5 Limit cycle oscillation corresponding to $\mu=0.5$
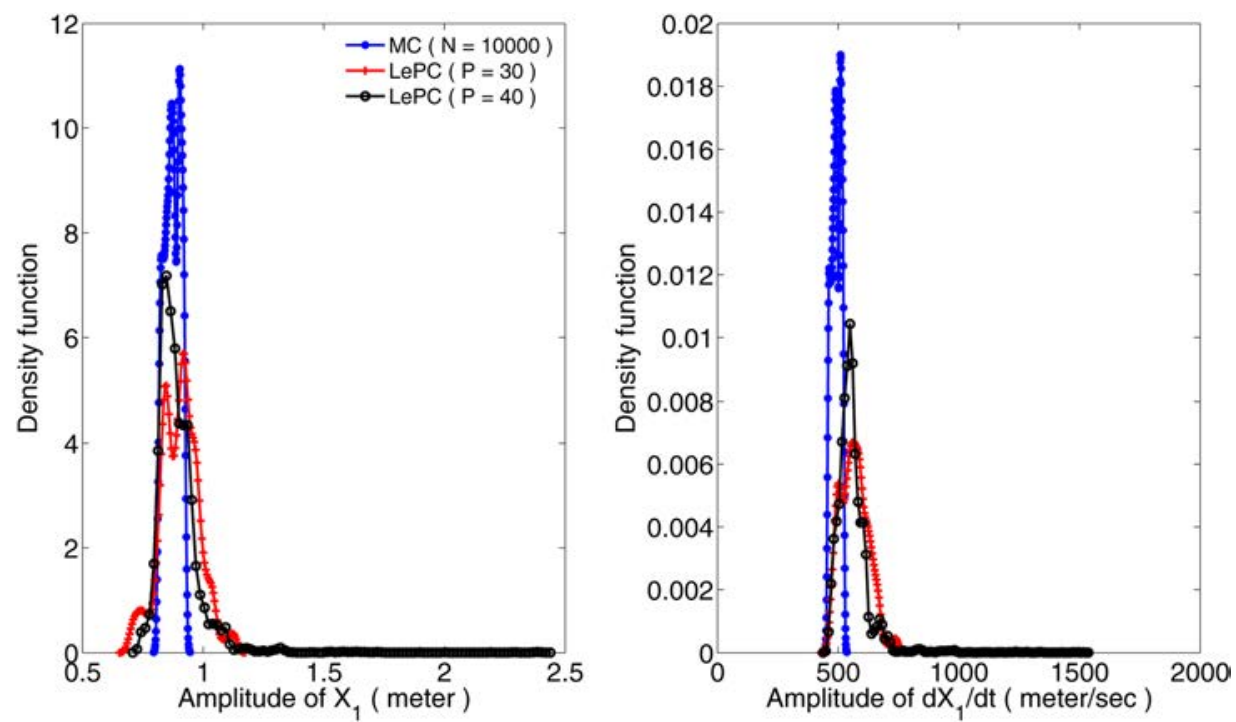

Fig. 6 Density functions of the LCO amplitude corresponding to the friction dispersion far from the Hopf bifurcation point estimated with the GPC based model

The friction coefficient at contact is assumed to be constant and the band moves at a constant velocity. Then it is assumed that the direction of the friction force does not change because the relative velocity between the band speed and $X_{1}$ or $X_{2}$ is assumed to be positive. The friction coefficient is assumed to be constant but uncertain. This uncertainty is generally related to the manufacturing process which yields uncertain tribological characteristics for the contact surfaces. For two manufactured systems, the corresponding friction coefficients are constant but with different values since the tribological characteristics are different.

The tangential force $F_{t}$ due to friction contact is assumed to be proportional to the normal force $F_{n}$ as given by Coulomb's law: $F t=\mu F_{n}$. Assuming that the normal $F_{n}$ is linearly related to the displacement of the mass normal to the contact surface, the resulting equations of motion can be expressed as [29-32].

$$
\dot{x}=f(x, \mu)=A(\mu) x+f_{N L}(x, \mu)
$$

where

$$
x=\left[\begin{array}{llll}
X_{1} & \dot{X}_{1} & X_{2} & \dot{X}_{2}
\end{array}\right]^{T}
$$

is the state vector,

$$
\begin{gathered}
A(\mu)=\left[\begin{array}{cccc}
0 & 1 & 0 & 0 \\
-\omega_{1}^{2} & -\eta_{1} \omega_{1} & \mu \omega_{2}^{2} & 0 \\
0 & 0 & 0 & 1 \\
-\mu \omega_{1}^{2} & 0 & -\omega_{2}^{2} & -\eta_{2} \omega_{2}
\end{array}\right] \\
f_{N L}(x, \mu)=\left[\begin{array}{c}
0 \\
-\varphi_{1}^{N L} X_{1}^{3}+\mu \varphi_{2}^{N L} X_{2}^{3} \\
0 \\
-\mu \varphi_{1}^{N L} X_{1}^{3}-\varphi_{2}^{N L} X_{2}^{3}
\end{array}\right]
\end{gathered}
$$

$\eta_{i}=c_{i} / \sqrt{M k_{i}}$ are the relative damping coefficients, $\omega_{i}=\sqrt{k_{i} / M}$ are the natural pulsations and $\varphi_{i}^{N L}=k_{i}^{N L} / M$ for $i=1,2$. For a numerical application: $M=1 \mathrm{Kg}, \omega_{1}=2 \pi \times 100 \mathrm{rad} / \mathrm{s}$, $\omega_{2}=2 \pi \times 75 \mathrm{rad} / \mathrm{s}, \eta_{1}=\eta_{2}=0.02, \varphi_{1}^{N L}=\omega_{1}^{2}$ and $\varphi_{2}^{N L}=0$.

The evolution of Eq. (22) linearized around its equilibrium point (origin, $x=0$ ) is plotted against the values of the friction coefficient in Fig. 2. The system has two pairs of conjugate eigenvalues. The evolution of real and imaginary parts of eigenvalues shows the well-known mode coupling phenomenon: with the 

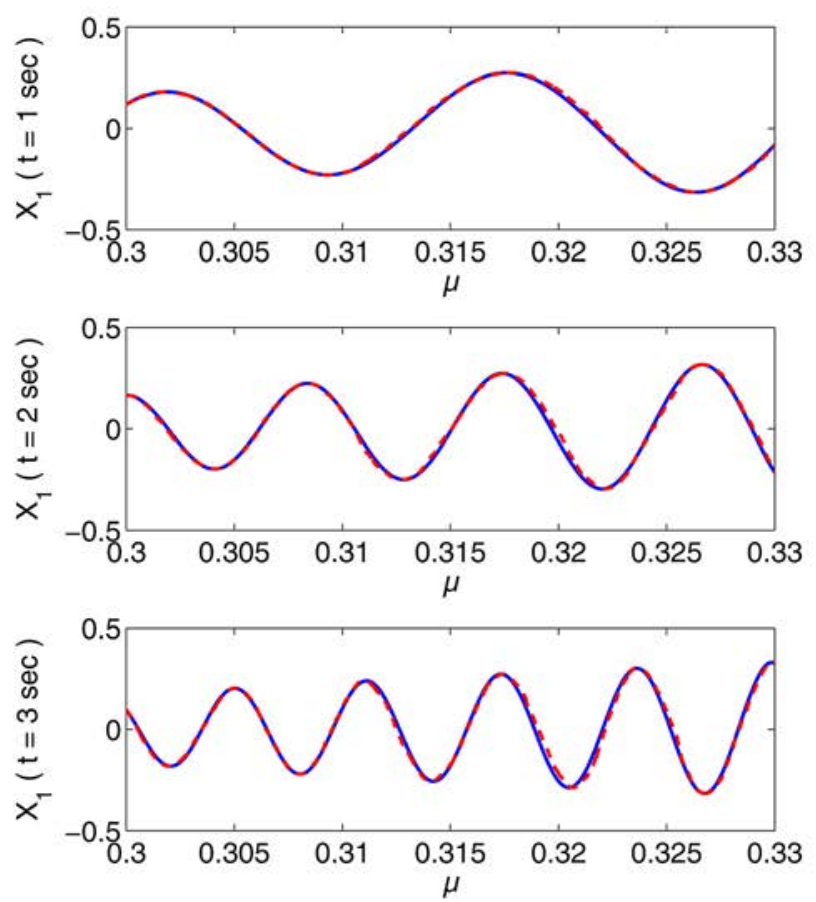
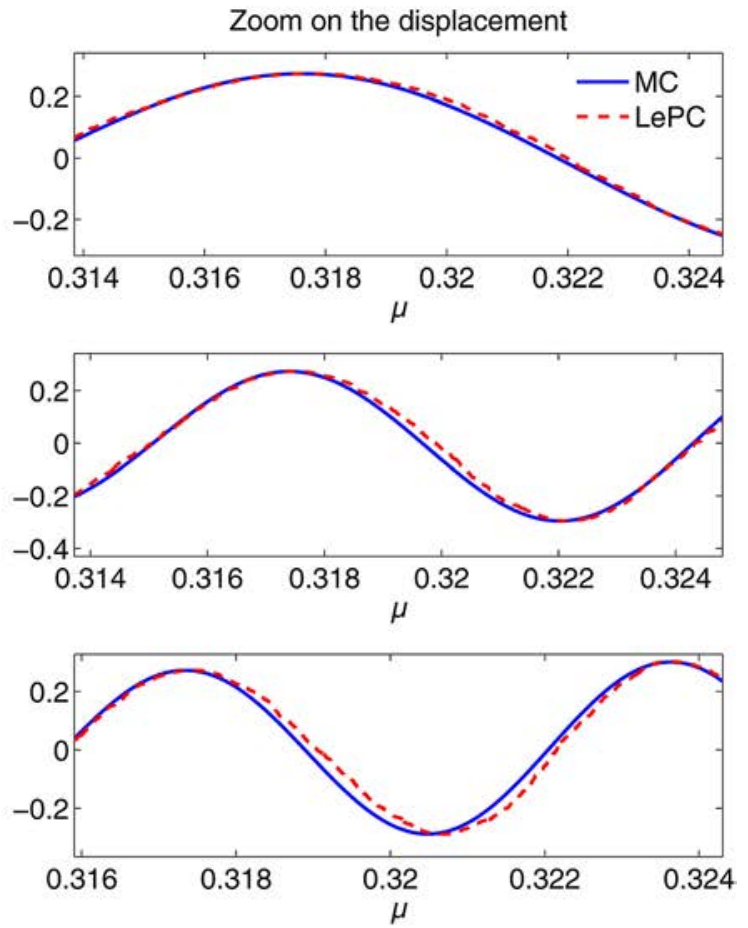

Fig. 7 The evolution of the displacement $X_{1}$ with respect to the friction coefficient in the zone near the Hopf bifurcation point, $[0.3,0.33]$
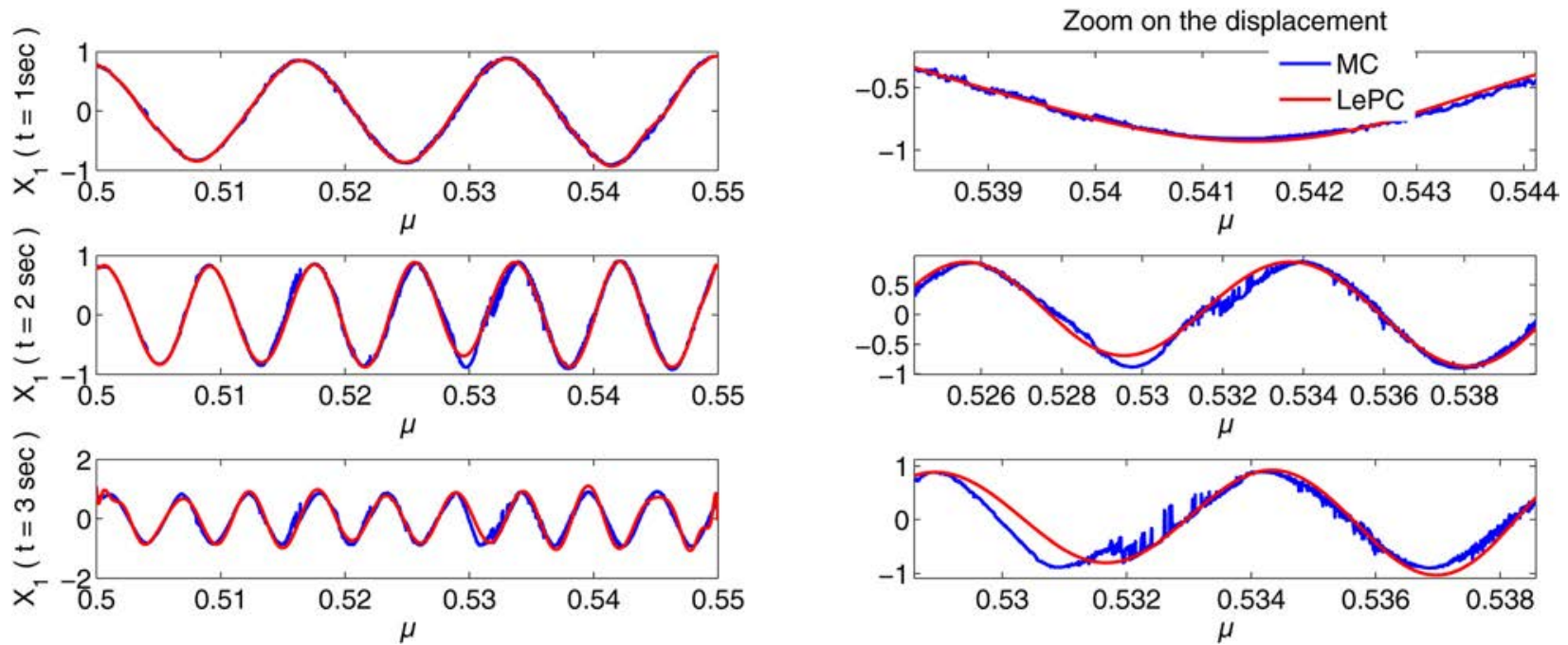

Fig. 8 The evolution of the displacement $X_{1}$ with respect to the friction coefficient in the zone near the Hopf bifurcation point, $[0.5,0.55]$

increase of the friction coefficient, the two modes of the system are close in frequency until coalescence in a particular point ( $\mu=\mu_{0} \approx 0.2894$ ) is called the Hopf bifurcation point. At this point, the two modes become equal while the corresponding real parts separate; one of the latter becomes positive. Consequently, the equilibrium position of the system becomes unstable for an infinitesimal perturbation and thus, self-friction induced vibrations occur. The main aim is to model and predict these oscillations and to estimate the dispersion of their amplitude when the friction dispersion is located near and far from the Hopf bifurcation point. The GPC, the ME-GPC, and the Wiener-Haar expansions are proposed to deal with these problems. The main idea is to express the system's states as polynomial chaos or Wiener-Haas wavelets expansions as given by Eqs. (3), (11), and (19).
The friction coefficient $\mu$ is uncertain and supposed to be driven by a uniform distribution law within the interval [0.3, 0.33]. Consequently, the dynamic behavior of Eq. (22) becomes stochastic. To predict its behavior according to the parameter requires the numerical resolution of Eq. (22). This operation is in general very difficult and costly due to complex nature of nonlinear dynamic systems. The modeling of Eq. (22) with the Wiener-Askey and Wiener-Haar expansions is analyzed below.

5.2 Modeling and Prediction of LCO Near the Hopf Bifurcation Point. The first problem is to model LCOs and to estimate the dispersion of their amplitude corresponding to a uniform friction coefficient within $[a, b]=[0.3,0.33]$ located near the Hopf 


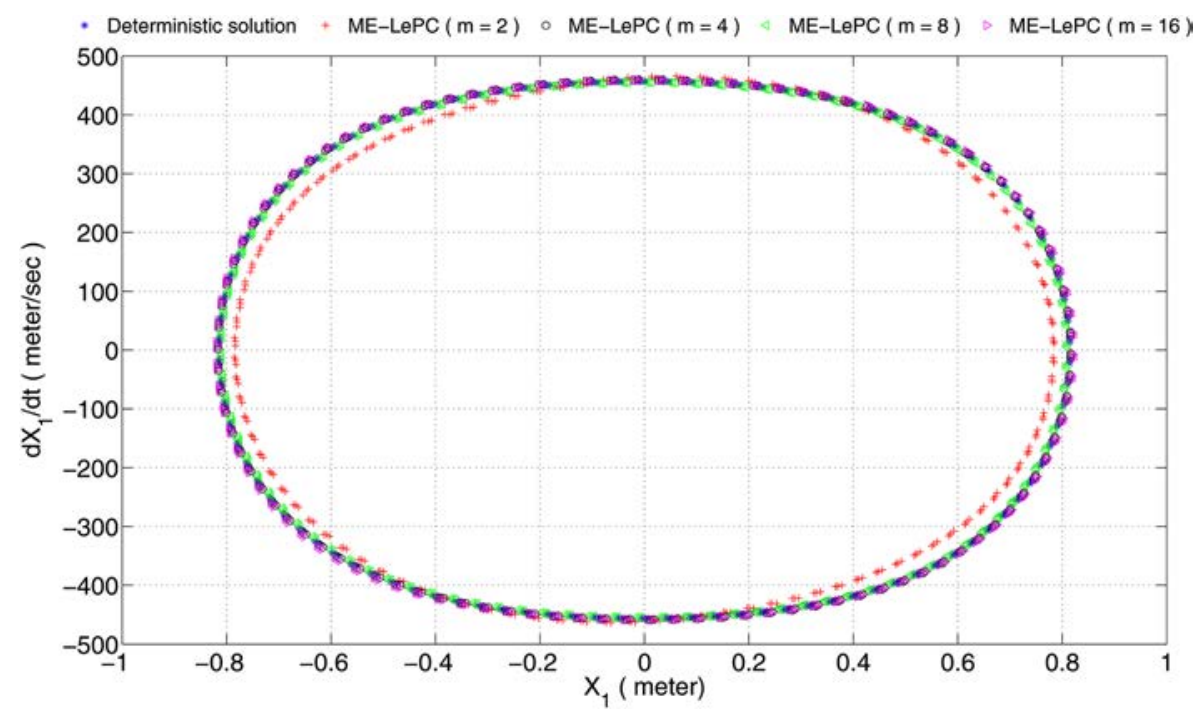

Fig. 9 Limit cycle oscillation corresponding to $\mu=0.5$ predicted with the ME-GPC model
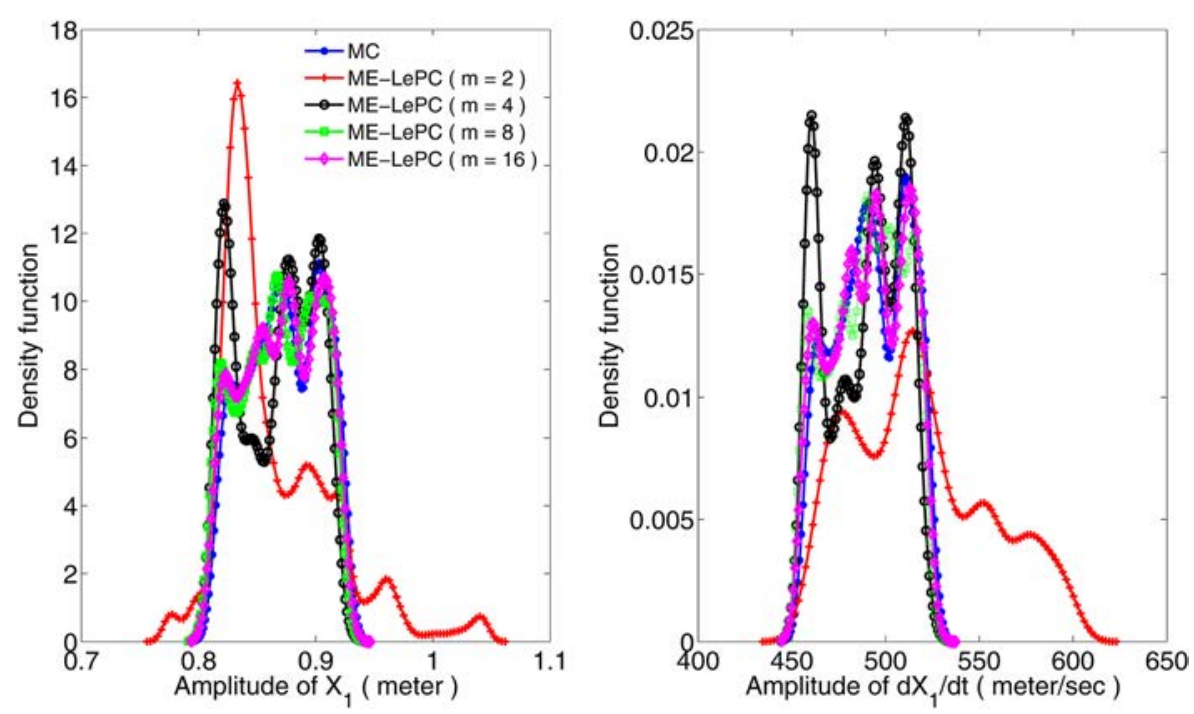

Fig. 10 Density functions of the LCO amplitudes corresponding to the friction dispersion far from the Hopf bifurcation point estimated with the ME-GPC based model

Table 3 Statistics of the amplitude of $X_{1}$ estimated with the ME-GPC far from the Hopf bifurcation

\begin{tabular}{lrrrrr}
\hline \hline CPG-ME & $m=2$ & $m=4$ & $m=8$ & $m=16$ & \multicolumn{1}{c}{ MC } \\
\hline Mean & 0.8650 & 0.8654 & 0.8683 & 0.8703 & 0.8730 \\
Std & 0.0485 & 0.0328 & 0.0321 & 0.0322 & 0.0316 \\
Min. & -1.0448 & -0.9202 & -0.9220 & -0.9263 & -0.9260 \\
Max. & 1.0448 & 0.9198 & 0.9208 & 0.9263 & 0.9260 \\
\hline \hline
\end{tabular}

bifurcation point $\mu_{m}=\mu_{0}(1+\varepsilon), \varepsilon=0.09$ with $\mu_{m}=0.315$ being the mean value of the friction dispersion and $\mu_{0} \approx 0.2894$ is the estimated Hopf bifurcation point. This problem has been dealt with in previous studies [29-32]. This study compares performances of the GPC (in particular, the Legendre polynomial chaos expansion (LePC)) and Wiener-Haar expansions.

Particular realizations of the displacement $x_{1}(t, \xi)$ approximated by the LePC and Wiener-Haar expansions are compared in Fig. 3 to the ones obtained from the solution of Eq. (22) by using the ODE MATLAB function. The LePC order $P$ and the resolution level $J$ are fixed, respectively, to $P=30$ and $J=7$, as shown in
Table 4 Statistics of the amplitude of $d X_{1} / \mathrm{dt}$ estimated with the ME-GPC far from the Hopf bifurcation

\begin{tabular}{lrrrrr}
\hline \hline ME-LePC & $m=2$ & $m=4$ & $m=8$ & $m=16$ & \multicolumn{1}{c}{ MC } \\
\hline Mean & 518.0657 & 488.2448 & 489.5521 & 490.6609 & 492.3341 \\
Std & 36.7938 & 20.0271 & 19.4584 & 19.5841 & 19.2087 \\
Min. & -602.1431 & -521.8708 & -521.2620 & -525.1518 & -524.1963 \\
Max. & 602.2645 & 521.6795 & 521.5239 & 525.2058 & 524.1963 \\
\hline \hline
\end{tabular}

previous studies $[29,32]$ to be the orders which gives the best LePC and Wiener-Haar approximations. Otherwise, the density functions of the amplitudes of the displacement $X_{1}$ and the corresponding velocity $\dot{X}_{1}$ are plotted in Fig. 4 . The Monte Carlo method using $N=10.000$ solutions of Eq. (22) helps to estimate the referential density functions while those estimated with the LePC and Wiener-Haar expansions are obtained by sampling, respectively, the random variables $\xi$ and $z$ and computing the corresponding sums. It is obvious that the MC procedure is too costly since the solutions from the LePC and Wiener-Haar expansions only require functions to be evaluated and summed. Note that the 

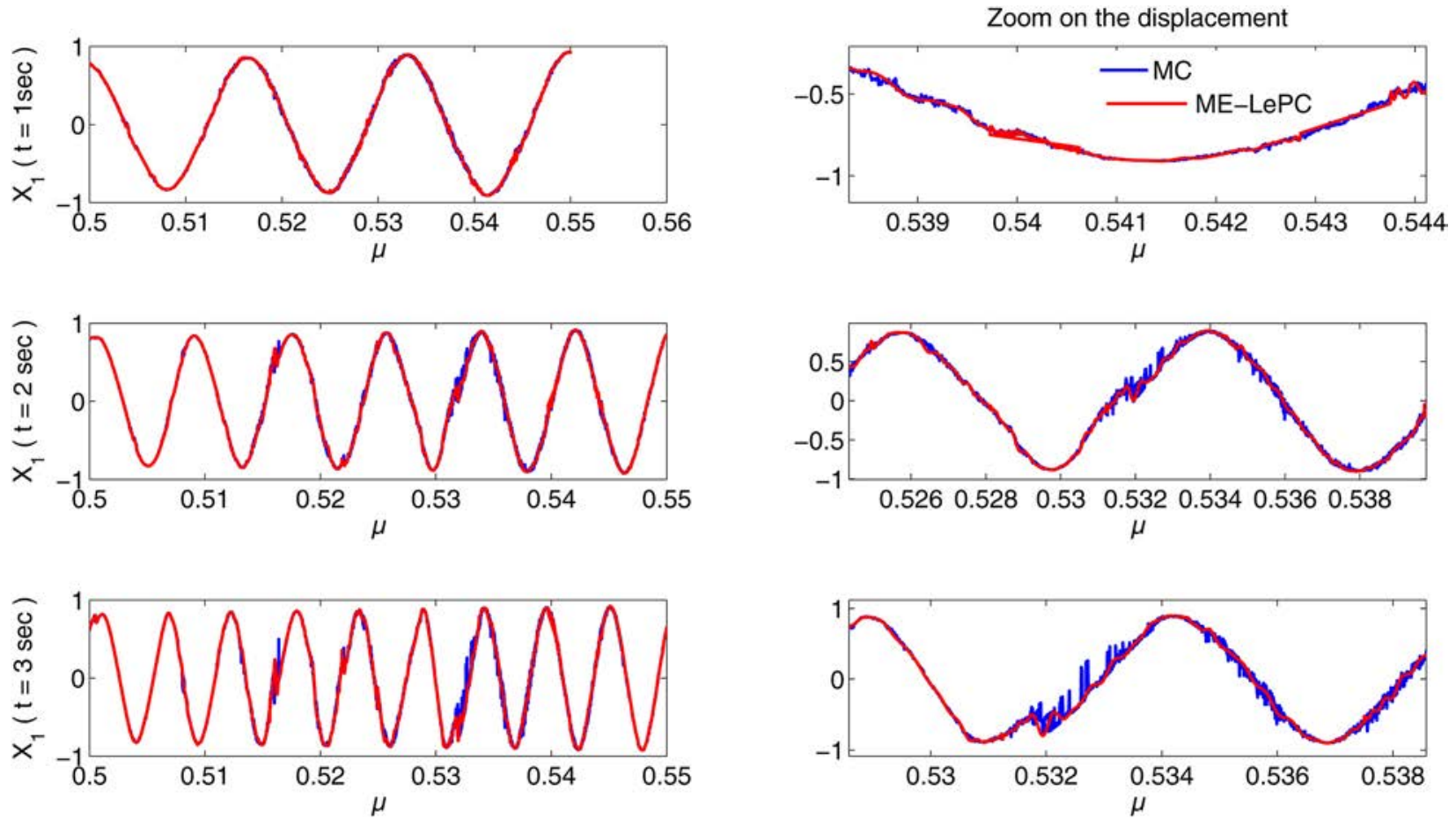

Fig. 11 The evolution of the displacement $X_{1}$ with respect to the friction coefficient in the zone near the Hopf bifurcation point, $[0.5,0.55]$ estimated with the ME-LePC

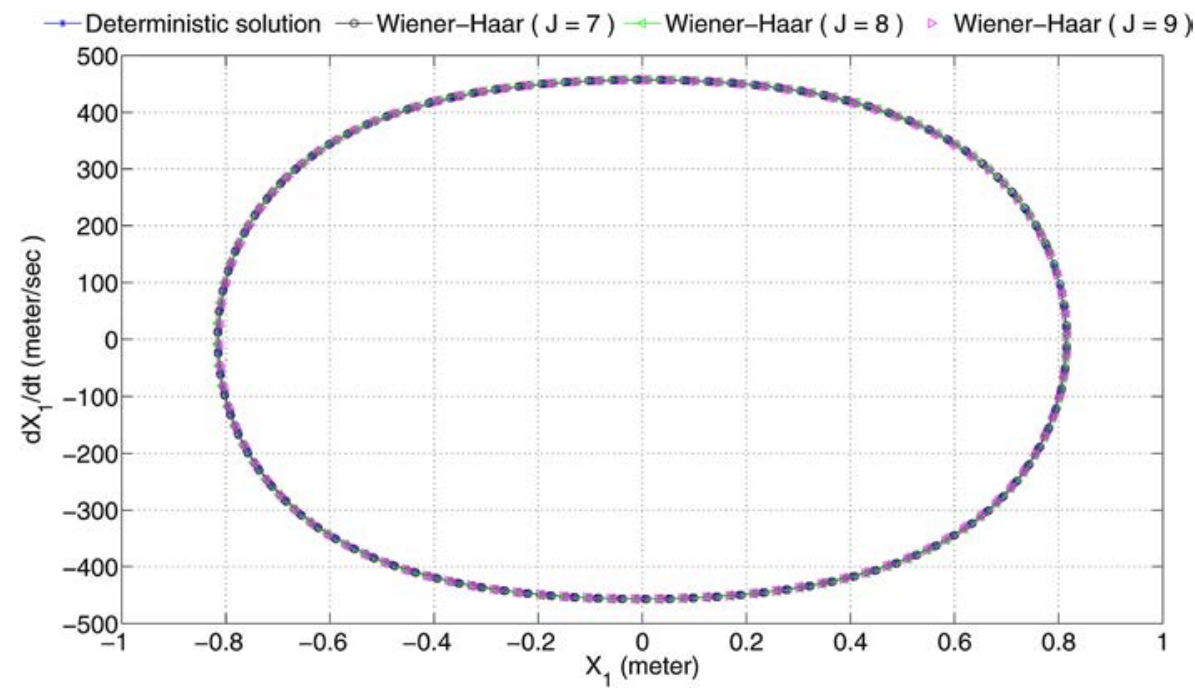

Fig. 12 Limit cycle oscillation corresponding to $\mu=0.5$ predicted with the Wiener-Haar expansion

number $N$ has been fixed to ensure high confidence and accuracy (99\% with error margin $1 \%)$.

From all the results, it can be noted that both GPC and the Wiener-Haar expansions model the referential LCOs obtained from the solution accurately, using the MATLAB ODE function of Eq. (22) with $\mu=0.3, \mu=0.315$, and $\mu=0.33$. GPC and Wiener- Haar expansions approximate the referential density functions of amplitudes of the displacement $X_{1}$ and the corresponding velocity $\dot{X}_{1}$ almost with the same accuracy. The corresponding statistics (mean value, standard deviation and global minimum and maximum) of the LCO amplitudes, given in Tables 1 and 2, confirm the high accuracy of both the GPC and Wiener-Haar expansions, MC results being the reference. However, it must be noted that the GPC is more efficient than the Wiener-Haar. Indeed, the computing of the GPC expansion using the regression technique has required only 35 solutions of Eq. (22), while the computing of the Wiener-Haar expansion by the Mallat algorithm has required 256 solutions.

5.3 Modeling and Prediction of LCOs Far From the Hopf Bifurcation Point. The main focus in Sec. 5.3 is to model LCOs and to estimate the dispersion of their amplitude corresponding to a uniform friction within $[\mathrm{a}, \mathrm{b}]=[0.5,0.55]$ located far from the Hopf bifurcation point $\mu_{m}=\mu_{0}(1+\varepsilon), \varepsilon=0.8$ with $\mu_{m}=0.525$. The frequency and amplitude of LCO in these flutter region are greater than those of LCOs near the Hopf bifurcation point. The 

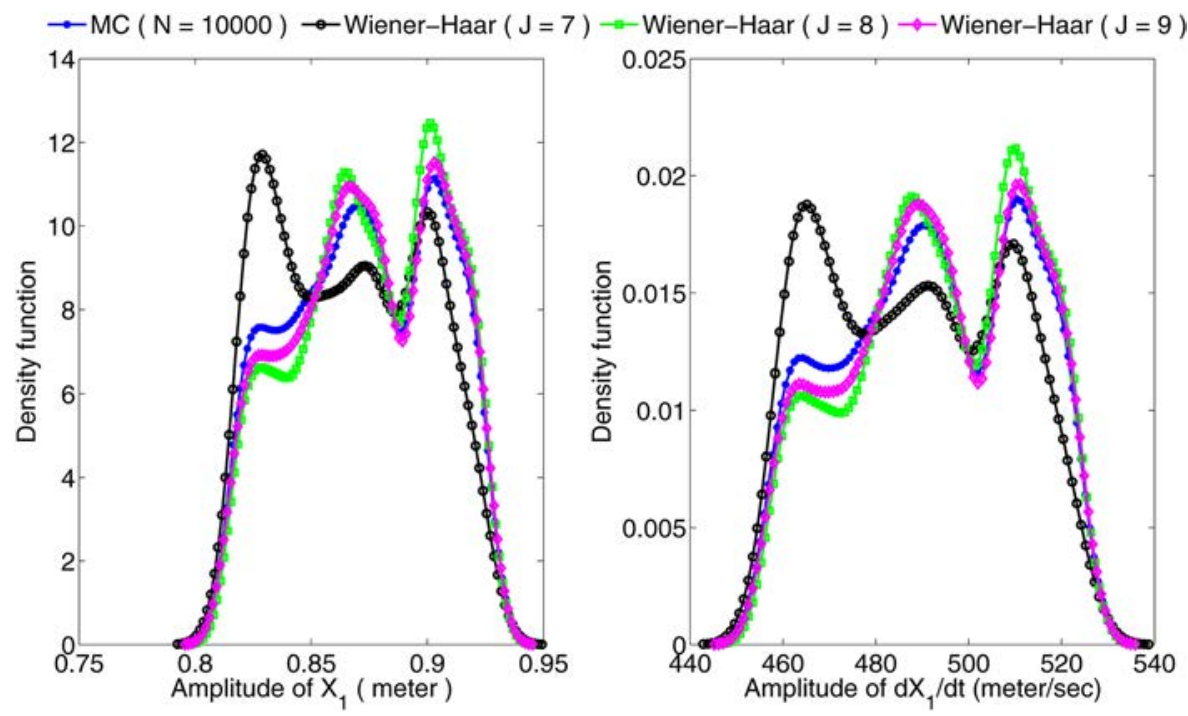

Fig. 13 Limit cycle oscillation corresponding to $\mu=0.5$ predicted with the Wiener-Haar expansion Density functions of the LCO amplitude corresponding to the friction dispersion far from the Hopf bifurcation point estimated with the Wiener-Haar based model

Table 5 Statistics of the amplitude of $X_{1}$ estimated with the Wiener-Haar model far from the Hopf bifurcation

\begin{tabular}{lrrrr}
\hline \hline Wiener-Haar & $J=7$ & \multicolumn{1}{c}{$J=8$} & $J=9$ & \multicolumn{1}{c}{ MC } \\
\hline Mean & 0.8666 & 0.8757 & 0.8743 & 0.8730 \\
Std & 0.0318 & 0.0308 & 0.0313 & 0.0316 \\
Min. & -0.9259 & -0.9257 & -0.9259 & -0.9260 \\
Max. & 0.9259 & 0.9257 & 0.9259 & 0.9260 \\
\hline \hline
\end{tabular}

Table 6 Statistics of the amplitude of $d X_{1} / d t$ estimated with the Wiener-Haar model far from the Hopf bifurcation

\begin{tabular}{lrrrr}
\hline \hline Wiener-Haar & \multicolumn{1}{c}{$J=7$} & \multicolumn{1}{c}{$J=8$} & \multicolumn{1}{c}{$J=9$} & \multicolumn{1}{c}{ MC } \\
\hline Mean & 488.4426 & 493.9819 & 493.1156 & \multicolumn{1}{c}{492.334} \\
Std & 19.4170 & 18.6994 & 18.9798 & 19.2097 \\
Min. & -524.1760 & -524.0464 & -524.1750 & -524.1963 \\
Max. & 524.1755 & 524.0464 & 524.1747 & 524.1963 \\
\hline \hline
\end{tabular}

objective is to check the capacities of GPC and Wiener-Haar expansions in the modeling and prediction of such LCOs.

5.3.1 Study With the GPC Expansion. First the 30th order GPC expansion is used, as it showed good accuracy in the first zone. As shown in Fig. 5, where one example of LCO corresponding to $\mu=0.5$ is plotted, the GPC expansion with $P=30$ computed by using the regression technique is not well suited to model LCOs in the considered flutter instability. The accuracy of the GPC expansion has not been enhanced significantly even with a significant increase of the GPC order $(P=40)$. The GPC expansion fails to well model and predict LCOs. This deficiency is also shown in Fig. 6 with the density functions of amplitudes of the displacement $X_{1}$ and the velocity $\dot{X}_{1}$. In order to explain how the GPC fails to well model LCO in flutter instability zone located far from the Hopf bifurcation point in contrast to the case when the flutter instability zone is near the Hopf bifurcation zone, the evolutions of the displacement $X_{1}$ with respect to the random friction coefficient in the two zones considered (near and far from the
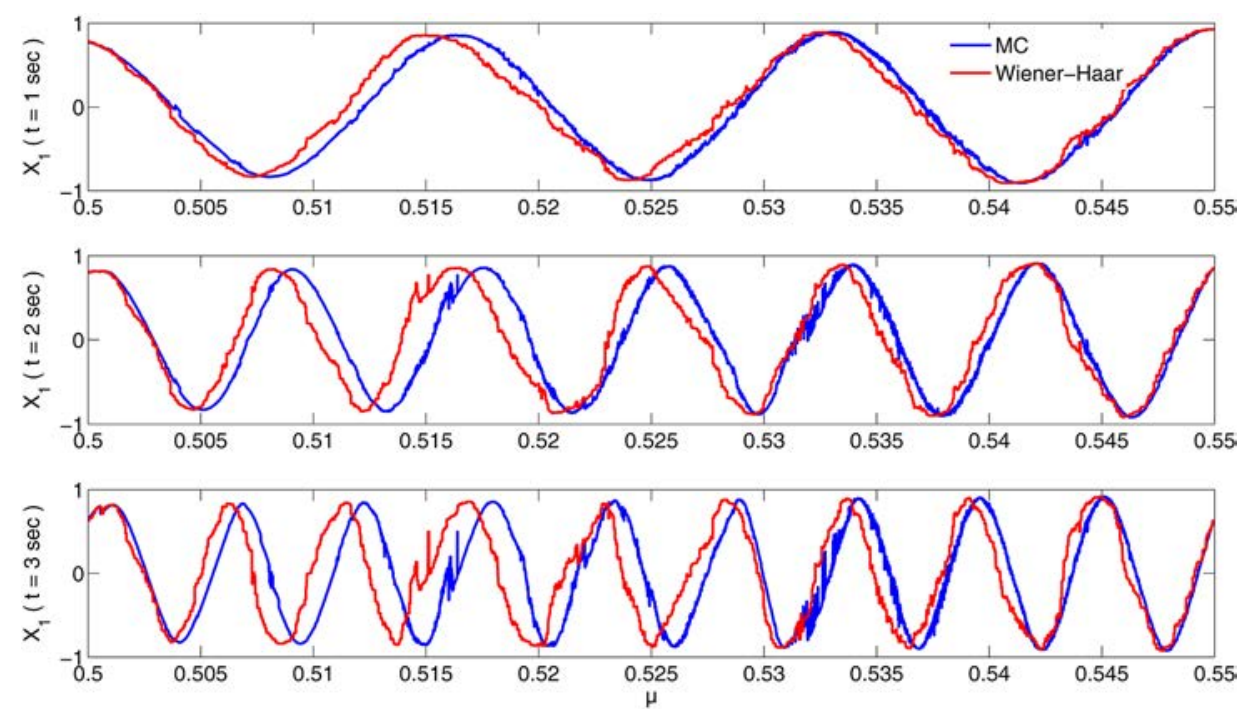

Fig. 14 The evolution of the displacement $X_{1}$ with respect to the friction coefficient in the zone near the Hopf bifurcation point, $[0.5,0.55]$ estimated with the Wiener-Haar expansion $(J=9)$ 
Hopf bifurcation point, [0.3, 0.33] and [0.5, 055]) are plotted at different times $t$, respectively, in Figs. 7 and 8 .

The evolution of the displacement $X_{1}$ in the random dimension estimated using the referential MC method is well approximated with the LePC expansion in the zone near the Hopf bifurcation point $([0.3,0.33]$, Fig. 7) in contrast to the zone far from the Hopf bifurcation point $([0.5,0.55]$, Fig. 8$)$. This can be justified by the incapability of the LePC expansion to well follow the variations of the displacement $X_{1}$ which become very fast and nonlinear as the distance of the uncertainty zone from the Hopf bifurcation point increases. The MC solution shows these fast and nonlinear variations in $[0.5,0.55]$.

The ME-GPC and Wiener-Haar expansions are proposed to circumvent the drawbacks of the LePC expansion.

5.3.2 Study With ME-GPC Expansion. Equation (16) is used with $P=30$ and different numbers $m$ of elements $(2,4,8,16)$. The LCO corresponding to $\mu=0.5$ estimated with this model is plotted in Fig. 9, while the density functions of LCO amplitudes are shown in Fig. 10.

The accuracy of the ME-LePC based model Eq. (16) increases with the number $m$ of elements. Good convergence properties with respect to $m$ are shown. The density functions of LCOs amplitudes (Fig. 10) and the corresponding statistics (mean value, standard deviation, minimum and maximum) (Tables 3 and 4) are suitably estimated in particular with $m=16$. With the latter, the maximum vibration levels are estimated with an error not exceeding $0.2 \%$ while the number of Eq. (22) solutions established for the computation of the ME-LePC model is 560. This is obviously much lower than the one required by the MC method $(N=10.000)$ fixed to have a $99 \%$ confidence interval with an error width about $1 \%$. Otherwise, the evolution of the displacement in the stochastic dimension (with respect to the friction coefficient) plotted in Fig. 11 shows the positive contribution of the ME-LePC based model with respect to the observed limits of the LePC expansion. Indeed, the ME-LePC model, in particular the one with $m=16$ plotted in Fig. 11, is better following the very fast, nonlinear variations of the displacement $X_{1}$ with the friction coefficient.

5.3.3 Study With the Wiener-Haar Expansion. Different resolution levels $J$ are considered for the Wiener-Haar based model Eq. (19) to find the most suitable resolution for the modeling and prediction of LCOs in the flutter instability zone [0.5, 0.55] situated far from the Hopf bifurcation point. The realization corresponding to $\mu=0.5$ is plotted in Fig. 12 for $J=7,8$, and 9. The same resolutions are used to plot the density functions of LCO amplitude in Fig. 13. The corresponding statistics are illustrated in Tables 5 and 6.

Contrary to the first study near the Hopf bifurcation point where the Wiener-Haar expansion with the $J=7$ was suitable to well model LCOs and the corresponding amplitudes, in the zone far from the Hopf bifurcation point, the same expansion is not sufficient. The resolution level required is higher, as shown in particular in the density functions plotted in Fig. 13. Indeed, to keep the same accuracy as in the study near the Hopf bifurcation point, it was necessary to increase the resolution level of the Wiener-Haar expansion until $J=9$. The latter gave more accurate estimations of both the density function of the LCO amplitudes and the corresponding statistics given in Table 5 and Table 6 . The maximum vibration amplitudes of LCOs are estimated with a relative error not exceeding $0.004 \%$, MC based solutions being the referential results obtained using $N=10,000$ solutions of Eq. (22) to ensure a high confidence level with a high accuracy $(99 \%$ with an error margin of $1 \%)$. For the same performances, it can be argued that only $\left(2^{J+1}<\mathrm{N}\right)$ solutions of Eq. (22) are required for the computation of the corresponding Wiener-Haar expansion. The contribution of the Wiener-Haar expansion can also be observed in the stochastic dimension. Indeed, as shown in Fig. 14, the expansion considered (in particular the one with $J=9$ ) models the very fast and nonlinear variations of the displacement $X_{1}$ in the random dimension better than the LePC expansion-with a small phase shift.

All the illustrated results show the efficiency of both the MELePC and Wiener-Haar expansions compared to the GPC in the modeling and prediction of LCOs when the dispersion of the friction coefficient is far from the Hopf bifurcation point. As the LePC expansion had shown good performance near the Hopf bifurcation point, it was not interesting to use the ME-LePC. The accuracy of the Wiener-Haar expansion is less sensitive at the location of the friction dispersion with respect to the Hopf bifurcation point.

\section{Conclusion}

This paper has been devoted to the modeling and prediction of friction induced vibrations in dry friction systems, taking account of the uncertainty of friction coefficients. The main aim was to assess the capacities of spectral expansions based on the Wiener chaos to well model and predict friction-induced limit cycle oscillations when the friction dispersion is located at different distance from the Hopf bifurcation point. It was interesting to consider this problem as the frequency and amplitude of friction-induced vibrations depend on the distance of the friction dispersion from the Hopf bifurcation point. The study compares the performances of GPC and Wiener-Haar expansion in the case the friction dispersion is located near the Hopf bifurcation point. Both expansions give suitable predictions of LCOs and the probabilistic dispersion of their amplitude. The GPC expansion proves to be more efficient, since for the same accuracy, it requires a smaller number of computations compared to the Wiener-Haar expansion. In the case the friction dispersion is located far from the Hopf bifurcation point, the GPC fails to well model and predict LCOs. In the zone considered, friction induced vibrations are characterized by LCOs with higher frequencies and amplitudes. The ME-GPC and the Wiener-Haar expansions are proposed to overcome GPC drawbacks. Indeed, the efficiency of the GPC based model is enhanced, using the Wiener-Haar expansion with a higher resolution level compared to the one used when the friction dispersion interval is near the Hopf bifurcation point. The efficiency of the GPC based model is also enhanced using the ME-GPC, which consists in dividing the friction dispersion interval into small elements and applying the GPC locally (in each element). There is a good compromise between cost and accuracy for both methods.

A more general conclusion can be stated about the more general use of the Wiener-Haar expansion compared to the GPC based model. The latter shows a higher sensitivity to the location of the friction dispersion with respect to the Hopf bifurcation point.

Finally, it can be recalled that this paper has been dedicated to the modeling and prediction of self-friction induced vibrations by taking into account the probabilistic dispersion of the friction coefficient supposed to be constant in accordance with the mode coupling phenomenon. In practice, other parameters hard to determine and thus uncertain should be taken into account. This is the case of stiffness, damping and mass which have a non-negligible effect on the dynamic behavior in particular on the vibration amplitudes in dry friction systems. As a result, it is necessary to take uncertainty of all these parameters into account in the modeling and prediction processes. This perspective is the aim of our research in progress.

\section{Acknowledgment}

The authors are grateful to the Agence Nationale (ANR) (French Research Agency) for its financial support.

\section{References}

[1] Ouyang, H., andMottershead, J. E., 2001, "Unstable Travelling Waves in the Friction-Induced Vibration of Discs,” J. Sound Vib., 248, pp. 768-779. 
[2] Sinou, J. J., Dereure, O., Mazet, F., Thouverez, F., and Jezequel, L., 2006, "Friction-Induced Vibration for an Aircraft Brake System-Part 1: Experimental Approach and Stability Analysis," Int. J. Mech. Sci., 48, pp. 536-554.

[3] Sinou, J. J., Thouverez, F., Jezequel, L., Dereure, O., Mazet, F., 2006, "Friction-Induced Vibration fFor an Aircraft Brake System-Part 2: NonLinear Dynamics," Int. J. Mech. Sci., 48, pp. 555-567.

[4] Chevennement-Roux, C., Dreher, T., Alliot, P., Aubry, E., Lainé, J. P., and Jézéquel, L., 2007, "Flexible Wiper System Dynamic Instabilities: Modeling and Experimental Validation," Exp. Mech., 47, pp. 201-210.

[5] Hervé, B., Sinou, J.-J., Mahé, H., and Jézéquel, L., 2009, "Extension of the dDestabilization Paradox to Limit Cycle Amplitudes for a Nonlinear SelfExcited System Subject to Gyroscopic and Circulatory Actions," J. Sound Vib., 323, pp. 944-973.

[6] Rudd, M. J., 1976, "Wheel/Rail Noise-Part II: Wheel Squeal," J. Sound Vib., 46, pp. 381-394.

[7] Coudeyras, N., Nacivet, S., and Sinou, J.-J., 2009, "Periodic and Quasi Periodic Solutions for Multi-instabilities Involved in Brake Squeal," J. Sound Vib., 328, pp. 520-540.

[8] Oberst, S., and Lai, J. C. S., 2011, "Statistical Analysis of Brake Squeal Noise," J. Sound Vib., 330(12), pp. 2978-2994.

[9] Oberst, S., and Lai, J. C. S., 2011, "Chaos in Brake Squeal Noise," J. Sound Vib., 330(5), pp. 955-975.

[10] Ibrahim, R. A., 1994, "Friction-Induced Vibration, Chatter, Squeal and Chaos-Part I: Mechanics of Contact and Friction," ASME Appl. Mech. Rev. 47, pp. 209-226.

[11] Ibrahim, R. A., 1994, "Friction-Induced Vibration, Chatter, Squeal and Chaos-Part II: Dynamics and Modeling," ASME Appl. Mech. Rev., 47, pp. 227-253.

[12] Spurr, R. T., 1961, “A Theory of Brake Squeal,” Proc. Inst. Mech. Eng., 1, pp. $33-40$.

[13] Earles, S. W. E., and Lee, C. K., 1976, "Instabilities Arising From the Frictional Interaction of a Pin- Disc System Resulting in Noise Generation," Trans. ASME, 1, pp. 81-86.

[14] Earles, S., and Chambers, P., 1987, "Disc Brake Squeal Noise Generation: Predicting Its Dependency on System Parameters Including Damping," Int. J. Veh. Des., 8, pp. 538-552.

[15] Herve, B., Sinou, J.-J., Mahe, H., and Jezequel, L., 2008, “Analysis of Squeal Noise and Mode Coupling Instabilities Including Damping and Gyroscopic Effects," Eur. J. Mech. A/Solids, 27, pp. 141-160.

[16] Antoniou, S. S., Cameron, A., and Gentle, C. R., 1976, "The Friction-Speed Relation From Stick-Slip Data," Wear, 36, pp. 235-254.

[17] Oden, J. T., and Martins, J. A. C., 1985, "Models and Computational Methods for Dynamic Friction Phenomena," Comput. Methods Appl. Mech. Eng., 52, pp. 527-634.

[18] Van De Velde, F., and De Baets, P., 1998, "A New Approach of Stick-Slip Based on Quasiharmonic Tangential Oscillations," Wear, 216, pp. 15-26.

[19] Van De Velde, F., and De Baets, P., 1998, "The Relation Between Friction Force and Relative Speed During the Slip-Phase of Stick-Slip Cycle," Wear, 219, pp. 220-226.

[20] Kinkaid, N., O'Reilly, O., and Papadopoulos, P., 2003, "Automotive Disc Brake Squeal,” J. Sound Vib., 267, pp. 105-166.

[21] Ouyang, H., Nack, W., Yuan, Y., and Chen, F., 2005, "Numerical Analysis of Automotive Disc Brake Squeal: A Review," Int. J. Vehicle Noise Vib., 1, pp. 207-231.

[22] Millner, N., 1978, "An Analysis of Disc Brake Squeal," SAE Technical Paper No. 780332.

[23] D'Souza, A. F., and Dweib, A. H., 1990, "Self-Excited Vibration Induced by Dry Friction-Part II: Stability and Limit-Cycle Analysis," J. Sound Vib., 137, pp. $177-190$.

[24] Eriksson, M., and Jacobson, S., 2001, "Friction Behaviour and Squeal Generation of Disc Brakes at Low Speeds," Proc. Inst. Mech. Eng., 215, pp. 1245-1256.
[25] Hoffmann, N., and Gaul, L., 2003, "Effects of Damping on Mode-Coupling Instability in Friction Induced Oscillations," ZAMM, 83, pp. 524-534.

[26] Meziane, A., and Baillet, L., 2010, "Nonlinear Analysis of Vibrations Generated by a Contact With Friction," Eur. J. Comput. Mech., 19(1-3), pp. 305-316.

[27] Ragot, P., Berger, S., and Aubry., E., 2008, "Interval Approach Applied to Blades of Windscreens Wiper," Int. J. Pure Appl. Math., 46(5), pp. 643-648.

[28] Lee, H. W., Sandu, C., and Holton, C., 2010, "Wheel-Rail Dynamic Model and Stochastic Analysis of the Friction in the Contact Path," ASME Conf. Proc/ 2010 Joint Rail Conference, Paper No. JRC2010-36229.

[29] Nechak, L., Berger, S., Aubry, E., 2011, "A Polynomial Chaos Approach to the Robust Analysis of the Dynamic Behavior of Friction Systems," Eur. J. Mech. A/Solids, 30(4), pp. 594-607.

[30] Nechak, L., Berger, S., and Aubry, E., 2013, "Non-Intrusive Generalized Polynomial Chaos for the Stability Analysis of Uncertain Dynamic Friction Systems," J. Sound Vib., 332(5), pp. 1204-1205.

[31] Nechak, L., Berger, S., and Aubry E., 2012, "Prediction of Random Self Friction Induced Vibrations in Uncertain Dry Friction Systems Using a MultiElement Generalized Polynomial Chaos Approach," J. Vibr. Acoust., 134(4), pp. 041015-041029.

[32] Nechak, L., Berger, S., and Aubry E., 2012, "Wiener-Haar Expansion for the Modelling and Prediction of the Dynamic Behaviour of Nonlinear Uncertain Systems," J. Dyn. Syst. Meas. Control, 134(5), pp. 051011-051022.

[33] Beran, P. S., Pettit, C. L., and Millman, D. L., 2006, "Uncertainty Quantification of Limit-Cycle Oscillations," J. Comput. Phys., 217, pp. 217-247.

[34] Wiener, N., 1938, “The Homogeneous Chaos," Am. J. Math., 60, pp. 897-936.

[35] Ghanem, R., Spanos, P. D., 1991, Stochastic Finite Elements: A Spectral Approach, Springer-Verlag, New York.

[36] Cameron, H., and Martin, W., 1947, "The Orthogonal Development of Nonlinear Functionals in Series of Fourier-Hermite Functional," Ann. Math., 48, pp 385-392.

[37] Xiu, D., and Karniadakis, G. E., 2002, "Modeling Uncertainty in Steady State Diffusion Problems via Generalized Polynomial Chaos," Comput. Methods. Appl. Mech. Eng., 191(43), pp. 4927-4948.

[38] Wan, X., and Karniadakis, G. E., 2006, "Beyond Wiener-Askey Expansions: Handling Arbitrary PDFs," J. Sci. Compt., 27(1-3), pp. 455-464.

[39] Babuska, I., Tempone, R., and Zouraris, G. E., 2004, "Galerkin Finite Element Approximation of Stochastic Elliptic Partial Differential Equations," SIAM J. Numer. Anal., 42(2), pp. 800-825.

[40] Babuska, I., Nobile, F., and Tempone, R., 2007, "A Stochastic Collocation Method for Elliptic Partial Differential Equations With Random Input Data,' SIAM J. Numer. Anal., 45(3), pp. 1005-1034.

[41] Crestaux, T., Le Maitre, O., and Martinez, J. M., 2009, "Polynomial Chaos Expansion for Sensitivity Analysis," Reliab. Eng. Syst. Saf., 94(7), pp. 1161-1172.

[42] Wan, X., and Karniadakis, G., 2005, "An Adaptive Multi-Element Generalized Polynomial Chaos Method for Stochastic Differential Equations," J. Comput. Phys., 209(2), pp. 617-642.

[43] Mallat, S., 1989, "A Theory for Multi-Resolution Signal Decomposition: The Wavelet Representation,” IEEE Trans. Pattern Anal. Mach. Intell., 11(8), pp. 674-694.

[44] Sinou, J.-J., and Jezequel, L., 2007, "Mode Coupling Instability in Friction Induced Vibrations and its Dependency on System Parameters Including Damping,” Eur. J. Mech. A/Solids., 26(1), pp. 107-122.

[45] Hultèn, J., 1993, "Drum Break Squeal-A Self-Exciting Mechanism With Constant Friction," Proceedings of the SAE Truck and Bus Meeting, SAE Paper No. 932965

[46] Sinou, J. J., Fritz, G., and Jezequel, L., 2007 "The Role of Damping and Definition of the Robust Damping Factor for a Self-Exciting Mechanism With Constant Friction,” J. Vib. Acoust., 129(3), pp. 297-307. 